\title{
Diagnosis of Giant Cell Tumor of the Tendon Sheath Using Multiecho Gradient-Echo Sequence: the "Superblooming Artifact"
}

\author{
Michele Gaeta, ${ }^{1}$ Giuseppe Cicero, ${ }^{1}$ Luca Fiorillo $\left(D,{ }^{1,2}\right.$ Sergio Vinci, ${ }^{1}$ Alfredo Blandino, ${ }^{1}$ \\ Gabriele Cervino $\left({ }^{\circ},{ }^{1}\right.$ Marco Cicciù $\left({ }^{\circ},{ }^{1}\right.$ and Fabio Minutoli ${ }^{1}$ \\ ${ }^{1}$ Department of Biomedical and Dental Sciences, Morphological and Functional Images, University of Messina, Messina 98100, Italy \\ ${ }^{2}$ Multidisciplinary Department of Medical-Surgical and Dental Specialties, University of Campania "Luigi Vanvitelli", \\ 80100 Napoli, Italy
}

Correspondence should be addressed to Luca Fiorillo; lucafiorillo@live.it

Received 13 October 2020; Accepted 4 March 2021; Published 17 March 2021

Academic Editor: Salvatore Cappabianca

Copyright (C) 2021 Michele Gaeta et al. This is an open access article distributed under the Creative Commons Attribution License, which permits unrestricted use, distribution, and reproduction in any medium, provided the original work is properly cited.

\begin{abstract}
Giant cell tumor of the tendon sheath is a tumor, which affects mainly the hands of people aged 30 to 50 years with a female prevalence. Magnetic resonance imaging (MRI) is essential for diagnosis and treatment planning. The aim of this study is to evaluate the efficacy of multiecho gradient-echoes (MeGE) sequence in detecting hemosiderin which is the hallmark of this tumor. MRIs were performed in a sample of 11 patients with a mean age of 45 . With the proposed protocol, all readers were able to detect the susceptibility artifacts due to the presence of hemosiderin. MeGE sequence allows to highlight the presence of hemosiderin, and the use of 3 echo times (ET) $(8,16$, and $24 \mathrm{~ms})$ is suggested.
\end{abstract}

\section{Introduction}

Giant cell tumor of the tendon sheath (GCTTS) is the second most common lesion of the hand after ganglion cysts $[1,2]$. The tumor affects subjects aged between 30 and 50 years old, with a female preponderance [3-7]. GCTTS is a slowly growing, usually painless benign lesion with a not trivial rate of recurrence (about $10 \%)$ and small risk to develop a malignant behavior $[8,9]$.

The microscopic features of GCTTS are foamy histiocytes, giant cells, large synovial cells, and hemosiderin deposits both intracellular and extracellular. Magnetic resonance imaging (MRI) is the most useful imaging modality for both diagnostic and surgical planning purposes [10], in particular, the MRI detection of intralesion artifacts due to hemosiderin deposits is the diagnostic hallmark of this tumor.

Gradient-echo (GE) sequences are the best diagnostic acquisitions in demonstrating the presence of hemosiderin, which appears as a loss of signal (the so-called blooming artifact). It is a susceptibility artifact, a distortion of the local magnetic field that makes small lesions more conspicuous [11-18].
However, to the best of our knowledge, no previous study has investigated the optimal GE parameters to use for improving hemosiderin detection. The aims of this work are the evaluation of a multiecho GE (MeGE) sequence effectiveness in detecting hemosiderin within GCTTSs and the assessment of the best echo time for GCTTS's diagnosis.

\section{Material and Methods}

2.1. Study Population. The present study retrospectively reviewed the MR images of 11 patients affected by surgically treated and histologically proven GCTTSs of the hands.

The 11 patients included two men and nine women, aged between 17 and 62 years (median 45 years). All patients manifested clinically as painless, soft, slow-growing tissue lumps without local redness, swelling, or ulceration. MRIs were performed over a 10-year period, between January 2010 and January 2020.

2.2. MR Examination. MRI was performed using a $1.5 \mathrm{~T}$ MRI scanner (Philips Achieva Philips Healthcare, Den Haag, the Netherlands). The scan parameters were as follows: T1weighted fast spin echo sequence (repetition time/echo time 
(TR/TE), $500 / 15 \mathrm{~ms}$; slice thickness, $3.0 \mathrm{~mm}$; and matrix scan, $256 \times 256)$ and T2 weighted turbo-spin echo sequence (TR/TE, 3000/90 ms; slice thickness, $3.0 \mathrm{~mm}$; field of view, 300-380 mm; and matrix scan, $256 \times 256$ ).

In all cases proton density-weighted imaging (PDWI; pulse sequences, TR/TE 2000-3500/20-30 (TR/TE)) fatsuppressed fast spin echo sequences and/or short-time inversion recovery (STIR) sequences (TR/TE 4500/40-50 ms, inversion time $150 \mathrm{~ms}$ ) were obtained (Table 1 ).

Fat-suppressed T1-w GE images with fat saturation were acquired after intravenous injection of $0.2 \mathrm{mmol} / \mathrm{kg}$ gadolinium-diethylene triamine pentaacetic acid (Dotarem, gadoterate meglumine, Guerbet, Roissy CdG Cedex-France) in all patients.

In addition, each patient was scanned with MeGE T2* sequence using the following parameters: flip angle $28^{\circ}$, TR $700^{\circ} \mathrm{ms}$, and echo times $8,16,24,32$, and $40^{\circ} \mathrm{ms}$. Slice thickness was 3-3.5 mm (Figures 1-4).

2.3. Images Analysis. After a previous anonymization and randomization process, $\mathrm{MR}$ images were evaluated by two radiologists (M.G., A.B., both with more than 20 years experience in MRI and musculoskeletal radiology), who were aware of the diagnosis of GCTTSs but were blind to the surgical findings.

The evaluation was divided in three different time points at intervals of one month to reduce bias due to memory of the previous analyses:

(i) First, only nonmultiecho GE (non-MeGE) were studied

(ii) Afterwards, only MeGE, in random order, were evaluated

(iii) Finally, MeGE images of each exam, ordered from lowest to highest echo time, were analyzed.

The readers were asked to assess the following items:

(1) Presence of hemosiderin in non-MeGE sequences (yes/no)

(2) Presence of hemosiderin in MeGE images, considering separately each echo time (yes/no)

(3) Confidence in distinguishing hemosiderin from fibrosis using increasing "blooming artifact" at different echo times (yes/no) on MeGE scans. This blooming increasing effect has been named "superblooming sign."

In addition, two different board-certified radiologists (G.C., S.M.) independently performed image quality assessment (good/inadequate) of MeGE acquisitions at each ET in a fourth separate session.

Image quality assessment was based on tumor signal intensity, identification of tumor edges, and low resolution due to susceptibility effect.

2.4. Statistics. The agreement of the two readers has been evaluated for each point 1,2, and 3 and for image quality.

\section{Results}

The agreement for detection of blooming between the two readers is summarized in Table 1 .

Non-MeGE images were not sensitive in detecting of blooming artifact. Only Reader 2 was confident enough to detect blooming on T2-weighted images in 2 patients. In all the cases, both readers were able to detect blooming on MeGE $24 \mathrm{~ms}, 32 \mathrm{~ms}$, and $40 \mathrm{~ms}$. The best echo comparison for superblooming was between GE $8 \mathrm{~ms}$ and GE $24 \mathrm{~ms}$ which allowed to detect this sign in $100 \%$ of the patients for both readers. For image quality, GE with ET at $32 \mathrm{~ms}$ and $40 \mathrm{~ms}$ were evaluated as inadequate by both readers due to large blooming artifact and low signal-tonoise ratio.

The lesion appears strongly hypointense in the $8 \mathrm{~ms}$ ET image. Also, the readers considered this image positive for the presence of blooming. However, both agreed that the clear increase of the blooming effect (superblooming) in higher ET images make the diagnosis more confident. For comparison, look at the collagen of the tendons which do not show superblooming effect making differential diagnosis between GGTTS and a possible fibrous containing tumor very reliable.

In addition, note that images at 32 and $40 \mathrm{~ms}$ are affected by a very low signal-noise ratio and by a large blooming artifact, both degrading the images in a severe way.

In the inset $\left(A^{\prime}\right)$, the presence of a subtle peripheral capsule (empty arrow) and thin fibrous septa (arrowheads) within the nodule could be appreciated. An area of slight low signal intensity can be seen in the left corner of the lesion (white arrow). Both the readers agreed that it was not possible to obtain the difference between fibrosis and blooming artifact. However, the other echoes show with greater advantage by increasing the blooming artifact in this area (superblooming) making diagnosis of hemosiderin deposition very confident. Note that the more ventral septa show a slight superblooming effect on images at 24, 32 , and $40 \mathrm{~ms}$, demonstrating that small amounts of hemosiderin can be detected only evaluating the presence of this sign.

\section{Discussion}

Magnetic susceptibility "blooming" artifact within GCTTS on GE images is related to the local magnetic field created by intra- and extracellular hemosiderin deposition [19]. Hemosiderin is a superparamagnetic material consisting of conglomerates of denatured proteins, lipids, and particles containing iron released by the breakdown of hemoglobin. Superparamagnetism is more closely related to ferromagnetism than to paramagnetism. GE scans are based on only a single RF pulse, with a flip angle lower than $90^{\circ}$, in combination with a readout gradient reversal. This results in short TR and TE and consequent fast scan times [20].

Hemosiderin causes intense spinning nuclei dephasing with a marked $\mathrm{T} 2 / \mathrm{T} 2 *$ shortening and a consequent dark appearance within accumulation areas. When longer TEs 
TABLE 1: MRI protocols.

\begin{tabular}{lcccccccccc}
\hline & T2 & T2 & STIR & T1Gd & GE8 & GE16 & GE24 & GE32 & GE40 & Superblooming \\
\hline Blooming presence 1 & 0 & 0 & 0 & 0 & 63.6 & 90.9 & 100 & 100 & 100 & 100 \\
Blooming presence 2 & 0 & 18.2 & 0 & 0 & 72.7 & 90.9 & 100 & 100 & 100 & 100 \\
Agreement & 100 & 81.8 & 100 & 100 & 90.9 & 100 & 100 & 100 & 100 & 100 \\
\hline
\end{tabular}

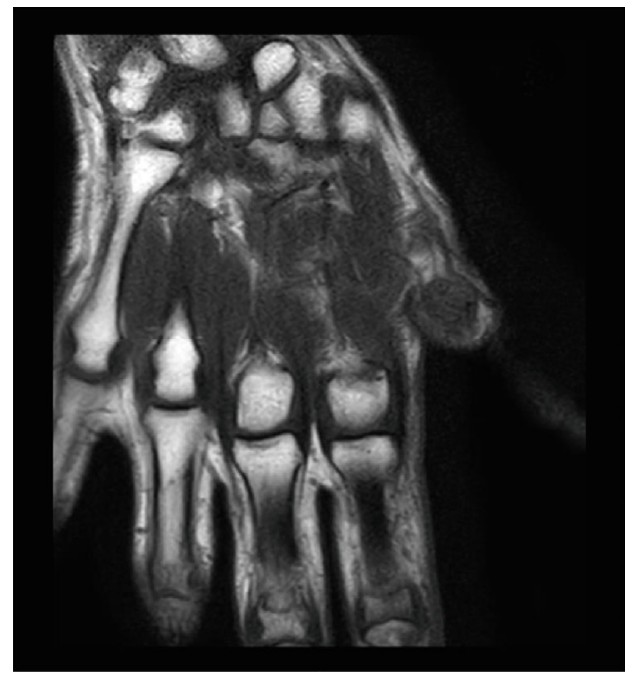

(a)

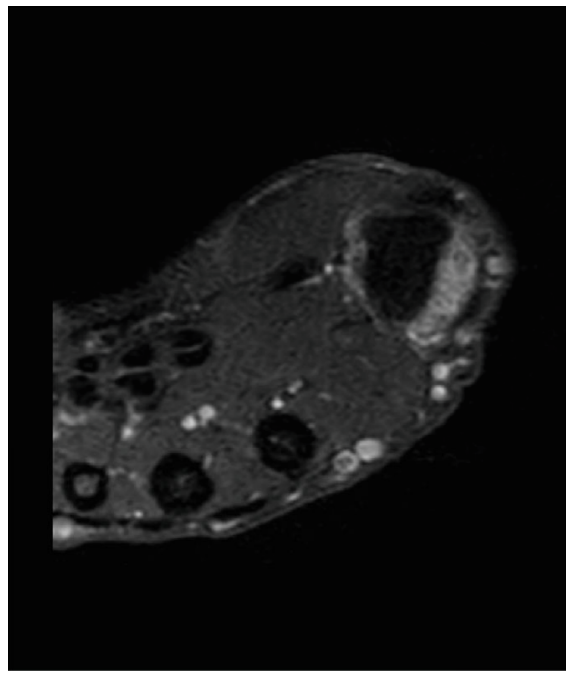

(b)

FIgURE 1: GCTTS with high hemosiderin content. Coronal T1-weighted scan (a) shows a nodule at the base of the I finger. On axial T1 fat-sat image (b), an intense but slight inhomogeneous enhancement of the lesion can be seen. No blooming can be seen in these sequences.

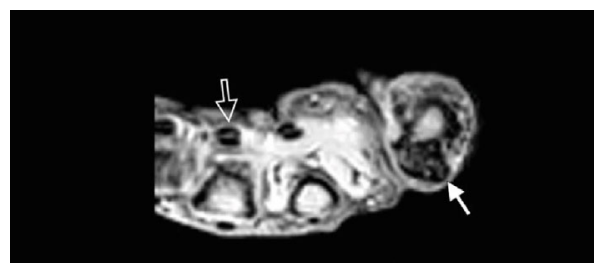

(a)

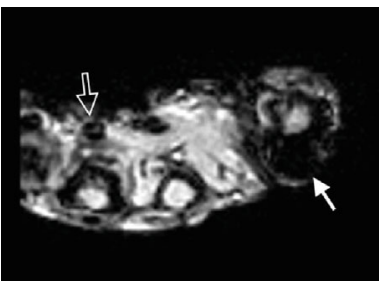

(c)

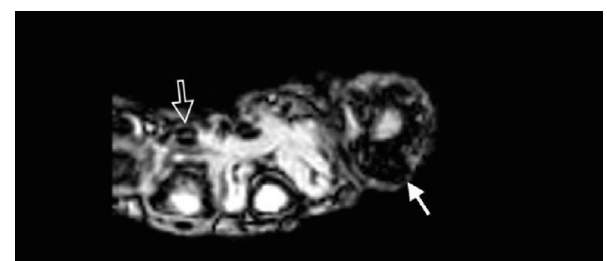

(b)

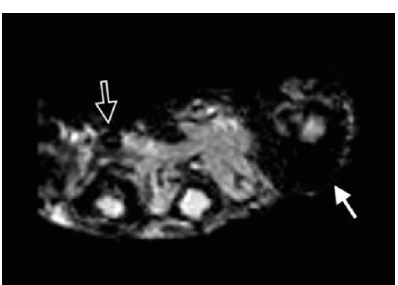

(d)

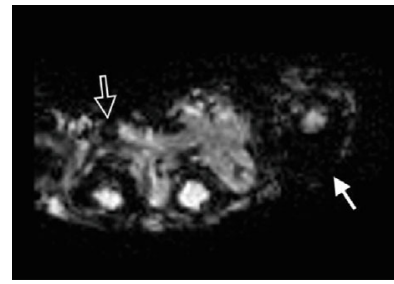

(e)

FIgURE 2: GCTTS with high hemosiderin content (same patient of Figure 1). Panel of five axial GE T2* images at different echo times, respectively, $8 \mathrm{~ms}$ (a), $16 \mathrm{~ms}$ (b), $24 \mathrm{~ms}$ (c), $32 \mathrm{~ms}$ (d), and $40 \mathrm{~ms}$ (e).

are used on GE scans, despite a general drop of signal intensity, a higher susceptibility to field inhomogeneity is determined, and therefore, hemosiderin deposits within GCTTSs can be easily detected [20].

However, the amount of hemosiderin is usually a much less prominent feature of tendon sheath disease than of its intra-articular counterpart also known as pigmented villonodular synovitis [21].
In addition, deposition variability of hemosiderin throughout the lesion is common in GCTTSs, which can contain foci of brighter T1 and T2 signals that indicate relatively low concentrations of hemosiderin.

On T2-weighted images, blooming may be difficult to appreciate, unless the amount of hemosiderin is massive. GCTTSs are surrounded by a collagen capsule, which spread into the tumor through septa dividing the lesion in lobules. 


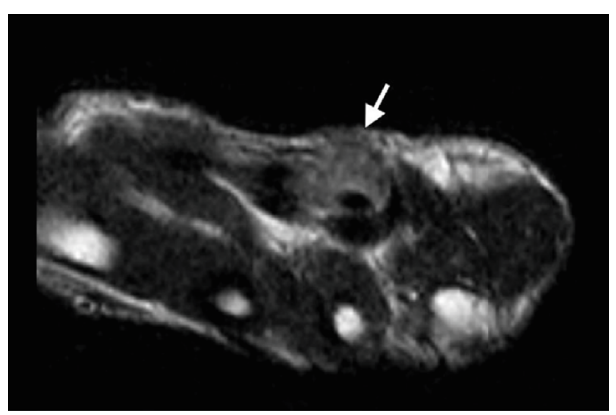

(a)

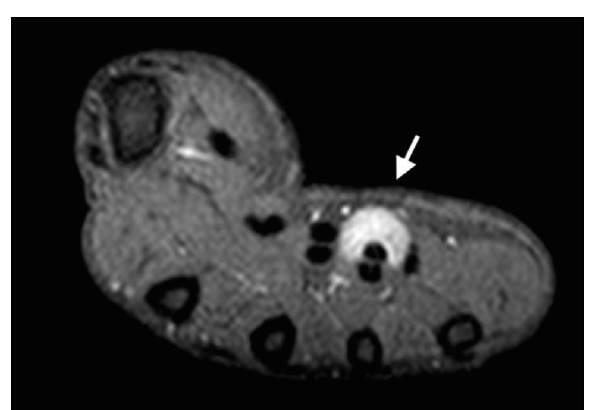

(b)

FIgURE 3: GCTTS with low hemosiderin content. AxialTSE T2 (a) and contrast-enhanced TSE T1 fat-sat (b) images through the palmar region of the hand show a nodular lesion involving the flexor tendons of the III finger. The lesion shows an intermediate signal on T2 weighted and an intense contrast-enhancement on postcontrast image. Both readers agreed on the absence of blooming artifact on both these images.

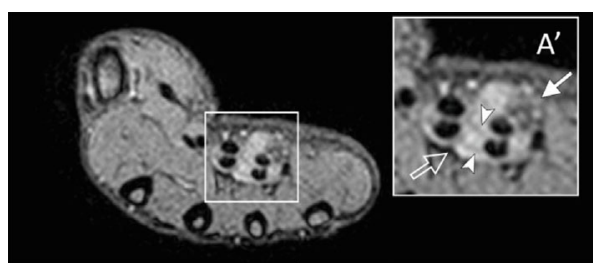

(a)

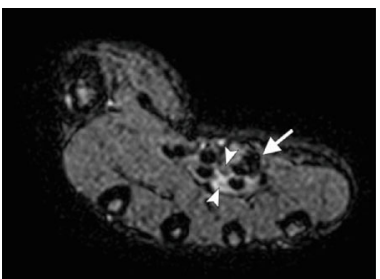

(c)

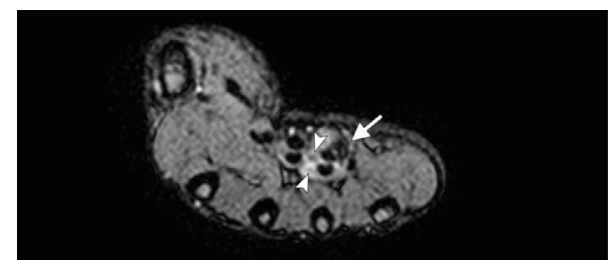

(b)

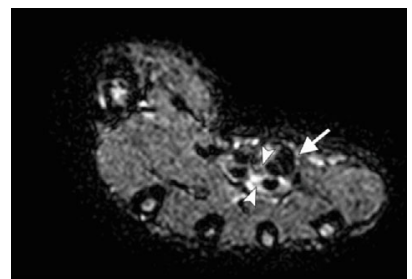

(d)

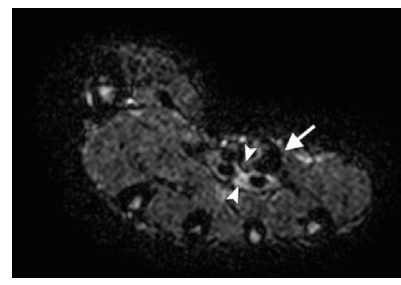

(e)

Figure 4: GCTTS with low hemosiderin content (same patient of Figure 3). Panel of five axial GE T2* images at different echo times, respectively, $8 \mathrm{~ms}$ (a), $16 \mathrm{~ms}$ (b), $24 \mathrm{~ms}$ (c), $32 \mathrm{~ms}$ (d), and $40 \mathrm{~ms}$ (e).

The presence of collagenous stroma can cause hypointensity within the tumor on MRI $[11,22]$.

The histological diagnosis of GCTTSs is rarely difficult but may be confused with features of other soft tissue tumors, particularly fibroma of the tendon sheath (FTS). Similarly, the clinical and imaging characteristics of FTS closely resemble those of GCTTSs.

Recently, a series including 18 patients with fibromas of the tendon sheath (FTS) and 24 with GCTTS was retrospectively evaluated by [24]. The authors found sensitivities, specificities, and diagnostic accuracies of $83-100 \%, 29-79 \%$, and $60-89 \%$, respectively, of MRI in differential diagnosis between these two entities.

However, the main drawback of this work is the lack of GE sequences in the MRI protocol. Since multiecho GE images can easily distinguish between fibrosis and hemosiderin, it could be supposed that differential diagnosis accuracy could reach $100 \%$ using this sequence. Nevertheless, considering that only GCTTs and not fibromas were evaluated in our work, further studies are necessary to confirm this hypothesis.

\section{Conclusion}

In conclusion, MeGE sequences allow detection of hemosiderin with great accuracy. The comparison between low ET and higher ET GE T2* sequences allowed in every case to confirm the presence of hemosiderin with complete agreement (high confidence) between readers. Since high ET GE $\mathrm{T} 2 *$ images beyond $24 \mathrm{~ms}$ showed a low image quality, the use of 3 ETs $(8,16$, and $24 \mathrm{~ms})$ is the better choice for assessment of suspected GCTTSs of the hands.

\section{Data Availability}

The manuscript data used to support the findings of this study are included within the article.

\section{Conflicts of Interest}

The authors declare that they have no conflicts of interest. 


\section{Authors' Contributions}

Conceptualization and writing original manuscript were done by M.G.; data curation was done by G.C.; writing review and editing were done by L.F.; investigation was done by S.V.; resources were acquired by A.B.; visualization was done by G.C.; resources were acquired by M.C.; and supervision was were done by F.M.

\section{References}

[1] F. M. Darwish and W. H. Haddad, "Giant cell tumour of tendon sheath: experience with 52 cases," Singapore Medical Journal, vol. 49, no. 11, pp. 879-882, 2008.

[2] I. J. F. Uriburu and V. D. Levy, "Intraosseous growth of giant cell tumors of the tendon sheath (localized nodular tenosynovitis) of the digits: report of 15 cases," The Journal of Hand Surgery, vol. 23, no. 4, pp. 732-736, 1998.

[3] S. S. Suresh and H. Zaki, "Giant cell tumor of tendon sheath: case series and review of literature," Journal of Hand and Microsurgery, vol. 2, no. 2, pp. 67-71, 2010.

[4] B. Garg and P. P. Kotwal, "Giant cell tumour of the tendon sheath of the hand," Journal of Orthopaedic Surgery, vol. 19, no. 2, pp. 218-220, 2011.

[5] H. Monaghan, D. M. Salter, and A. Al-Nafussi, "Giant cell tumour of tendon sheath (localized nodular tenosynovitis): clinicopathological features of 71 cases," Journal of Clinical Pathology, vol. 54, no. 5, pp. 404-407, 2001.

[6] E. L. Adams, E. M. Yoder, and M. L. Kasdan, "Giant cell tumor of the tendon sheath: experience with 65 cases," Eplasty, vol. 12, 2012.

[7] S. Di Grazia, G. Succi, F. Fraggetta, and R. E. Perrotta, "Giant cell tumor of tendon sheath: study of 64 cases and review of literature," Il Giornale di Chirurgia, vol. 34, no. 5-6, pp. 149-152, 2013.

[8] J. Williams, A. Hodari, P. Janevski, and A. Siddiqui, "Recurrence of giant cell tumors in the hand: a prospective study," The Journal of Hand Surgery, vol. 35, no. 3, pp. 451-456, 2010.

[9] J. G. Guccion, "Enzinger FM Malignant giant cell tumor of soft parts. An analysis of 32 cases," Cancer, vol. 29, no. 6, pp. 15181529, 1972.

[10] F. Gouin and T. Noailles, "Localized and diffuse forms of tenosynovial giant cell tumor (formerly giant cell tumor of the tendon sheath and pigmented villonodular synovitis)," Orthopaedics \& Traumatology, Surgery \& Research, vol. 103, no. 1, pp. S91-S97, 2017.

[11] J. M. Wan, N. Magarelli, W. C. G. Peh, G. Guglielmi, and T. W. H. Shek, "Imaging of giant cell tumor of the tendon sheath," $L a$ Radiologia Medica, vol. 115, no. 1, pp. 141-151, 2010.

[12] J. Jelinek, "Giant cell tumor of the tendon sheath: MR findings in nine cases," American Journal of Roentgenology, vol. 162, no. 4, pp. 919-922, 1994.

[13] A. Capelastegui, E. Astigarraga, G. Fernandez-Canton, I. Saralegui, J. A. Larena, and A. Merino, "Masses and pseudomasses of the hand and wrist: MR findings in 134 cases," Skeletal Radiology, vol. 28, no. 9, pp. 498-507, 1999.

[14] Y. Kitagawa, H. Ito, Y. Amano, T. Sawaizumi, and T. Takeuchi, "MR imaging for preoperative diagnosis and assessment of local tumor extent on localized giant cell tumor of tendon sheath," Skeletal Radiology, vol. 32, no. 11, pp. 633-638, 2003.
[15] A. Iovane, M. Midiri, T. V. Bartolotta et al., "Pigmented villonodular synovitis of the foot: MR findings," La Radiologia Medica, vol. 106, no. 1-2, pp. 66-73, 2003.

[16] J. Lin, J. A. Jacobson, D. A. Jamadar, and J. H. Ellis, "Pigmented villonodular synovitis and related lesions: the spectrum of imaging findings," American Journal of Roentgenology, vol. 172, no. 1, pp. 191-197, 1999.

[17] R. H. Dorwart, H. K. Genant, W. H. Johnston, and J. M. Morrisì, "Pigmented villonodular synovitis of synovial joints: clinical, pathologic, and radiologic features," American Journal of Roentgenology, vol. 143, no. 4, pp. 877-885, 1984.

[18] J. Llauger, J. Palmer, N. Rosón, R. Cremades, and S. Bagué, "Pigmented villonodular synovitis and giant cell tumors of the tendon sheath: radiologic and pathologic features," American Journal of Roentgenology, vol. 172, no. 4, pp. 1087-1091, 1999.

[19] R. Bitar, G. Leung, R. Perng et al., "MR pulse sequences: what every radiologist wants to know but is afraid to ask," Radiographics, vol. 26, no. 2, pp. 513-537, 2006.

[20] M. Markl and J. Leupold, "Gradient echo imaging," Journal of Magnetic Resonance Imaging, vol. 35, no. 6, pp. 1274-1289, 2012.

[21] M. D. Murphey, J. H. Rhee, R. B. Lewis, J. C. Fanburg-Smith, D. J. Flemming, and E. A. Walker, "Pigmented villonodular synovitis: radiologic-pathologic correlation," Radiographics, vol. 28 , no. 5, pp. 1493-1518.

[22] L. De Beuckeleer, A. De Schepper, F. De Belder et al., "Magnetic resonance imaging of localized giant cell tumour of the tendon sheath (MRI of localized GCTTS)," European Radiology, vol. 29, pp. 3441-3449, 2019. 